\title{
Interactive comment on "What can we learn about urban air quality with regard to the first outbreak of the COVID-19 pandemic? A case study from Central Europe" by Imre Salma et al.
}

Imre Salma et al.

salma@chem.elte.hu

Received and published: 9 December 2020

Dear Colleague,

we would like to thank you for this comment, which was adopted into the new MS.

Imre Salma corresponding author

Interactive comment on Atmos. Chem. Phys. Discuss., https://doi.org/10.5194/acp-2020-997, 2020. 